\title{
Perimeter of the Elliptical Arc a Geometric Method
}

\author{
Aravind Narayan
}

\begin{abstract}
There are well known formulas approximating the circumference of the Ellipse given in different periods in history. However there lacks a formula to calculate the Arc length of a given Arc segment of an Ellipse. The Arc length of the Elliptical Arc is presently given by the Incomplete Elliptical Integral of the Second Kind, however a closed form solution of the Elliptical Integral is not known. The current solution methods are numerical approximation methods, based on series expansions of the Elliptical Integral. This paper introduces a Geometric Method (procedure) to approximate the Arc length of any given Arc segment of the ellipse. An analytical procedure of the defined geometric method is detailed.

For a Circular Arc the Arc length of the Circular Segment is given through angular relations. However a similar formula for the Arc length of the Ellipse, using known trigonometric Identities is not known. The paper translates the defined geometric procedure to analytical, algebraic method using the known trigonometric identities. This method thus gives a method to estimate the Incomplete Elliptical Integral of the Second Kind using trigonometric identities.

The formulae for Circular Arc can be considered to be based on the intuitive reasoning "Equal Arcs cuts Equal Angles at the center for a Circle”. This paper presents a similar intuitive logic for the Elliptical Arc. The proposal is based on a geometric discovery based on intuitive reasoning and the algebraic theory explaining the intuitive logic is as such not yet developed. An analytical research into the intuitive logic behind the geometric approximation shall result in the development of the general theory and an analytical, algebraic proof of the proposed method.
\end{abstract}

\section{Introduction}

Let us define the Ellipse in the Canonical Position (Major Axis aligned with the $\mathrm{x}$ - axis, and the Center at the origin). Let the Major Axis length be '2a' and Minor Axis length be '2b'. The Semi Major Axis (Major Radius) is then ' $\mathbf{a}$ ' and the Semi Minor Axis (Minor Radius) is then ' $\mathbf{b}$ '.

The equation of the Ellipse in parametric form is given as

$$
\begin{aligned}
& x(t)=a \cos (t) \\
& y(t)=b \sin (t)
\end{aligned}
$$

The parameter ' $t$ ' is called the eccentric anomaly and it is not the angle between the Radius and the $\mathrm{x}$-axis. Intent

Our intent is to determine the Arc length $\left(\boldsymbol{L}_{\boldsymbol{e}}\right)$ of the given Elliptical Arc Segment AB (Perimeter of the Elliptical Arc AB) lying within a Quadrant of the Ellipse.

\section{The Conjure}

In order to determine the Arc Length of the Elliptical Arc we transform the Elliptical Arc to a Circular Arc such that the Circular Arc has the same Arc length as that of the Elliptical Arc. The following Conjure defines this Equivalent Circular Arc.

"The Equivalent Circular Arc having the same Arc length as that of a given Elliptical Arc segment (within a Quadrant Arc), will have a Chord length equal to the Chord length of the given Elliptical Arc and it (Circular Arc) will subtend an angle at the center whose value in radians is equal to the difference in the Eccentric anomalies of the end points of the given Elliptical Arc"

From the above conjure we can define a geometric procedure for determining the Arc length of the Ellipse.

\section{The Geometric Procedure for Determining the Arc Length}

Procedure

$>$ Identify the coordinates of the End points ' $\mathbf{A}$ ' and 'B' of the given Elliptical Arc Segment

$>$ Draw a Chord between the two End points 'A' \& 'B' of the Elliptical Arc segment .i.e. Chord AB

$>$ Determine the length of this Chord AB

$>$ Determine the Eccentric Anomalies (Parametric Values) of the End points ' $\mathbf{A}$ ' and ' $\mathbf{B}$ ' of the given Elliptical Arc Segment

$>$ Determine the difference between the Eccentric Anomaly Values as angle ' $\square$ ' in Radians.

$>$ Construct a Circular Arc whose Chord length is equal to the Chord length AB of the given Elliptical Arc and Subtending an angle ' $\square$ ' equal to the difference between the Eccentric Anomaly Values of the end 
points of the Elliptical Arc. Now for constructing this Circular Arc, the length of the radius of the Circular Arc can be determined by the following procedure

* Construct an isosceles triangle whose base length is equal to the length of the Chord of the Elliptical Arc, and the Angle opposite to the base is ' $\square$ '. The two equal angles are then $\left(\frac{\pi-\theta}{2}\right)$. (All angles in radians)

* Determine the length of the equal side of this Isosceles triangle, and Radius of the Circular Arc is equal to this length of the equal side of the Isosceles triangle

D The Arc length of this Circular Arc is equal to the Arc length of the Elliptical Arc.

In the following Section an Analytical Method is detailed giving the Analytical application of the Geometric Procedure.

\section{Analytical Procedure and Formula}

Identify the coordinates of the End points

Let us consider an Arc formed by two points ' $\mathbf{A}$ ' and ' $\mathbf{B}$ ' lying in the first Quadrant. Let the Coordinates of the point ' $\mathrm{A}$ ' be $\left(\boldsymbol{x}_{1}, \boldsymbol{y}_{1}\right)$ and that of ' $\mathbf{B}$ ' be $\left(\boldsymbol{x}_{2}, \boldsymbol{y}_{2}\right)$. Let the Point ' $\mathrm{A}$ ' have the parametric value ' $\boldsymbol{t}{ }_{\boldsymbol{1}}$ ' and Point ' $\mathbf{B}$ ' have the parametric value ' $\boldsymbol{t}_{\mathbf{2}}$ ', then the coordinates values of ' $\mathbf{A}$ ' and ' $\mathbf{B}$ ' are

$$
\begin{gathered}
x_{1}=a \operatorname{Cos}\left(t_{1}\right), \quad y_{1}=b \operatorname{Sin}\left(t_{1}\right) \& \\
x_{2}=a \operatorname{Cos}\left(t_{2}\right), \quad y_{2}=b \operatorname{Sin}\left(t_{2}\right)
\end{gathered}
$$

The Elliptical Arc AB

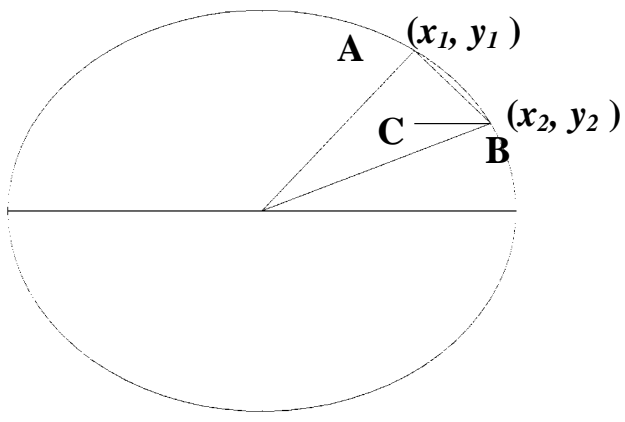

(Fig 1)

Chord Length $\mathbf{A B}$ can be determined by constructing the Right triangle $\mathbf{A B C}$. The chord $\mathbf{A B}$ forms the hypotenuse of the Right triangle $\mathbf{A B C}$. Side $\mathbf{A C}$ is given by the differential ' $\mathrm{x}$ '-coordinate length, and Side $\mathbf{B C}$ is given by the differential ' $y$ '-coordinate lengths, of the End points 'A: $\left(x_{1}, y_{1}\right)$ ' \& 'B: $\left(x_{2}, y_{2}\right)$ ' of the given Elliptical Arc

$$
\begin{gathered}
\mathrm{AC}=\Delta x=x_{2^{-}} x_{1} \\
\mathrm{BC}=\Delta y=y_{2}-y_{1} \\
\boldsymbol{h}=\mathbf{A B}=\sqrt{(\Delta \boldsymbol{x})^{2}+(\Delta \boldsymbol{y})^{2}}
\end{gathered}
$$

\section{Equivalent Circular Arc}

The Equivalent Circular Arc will have a Chord length equal to AB. Now for a Circular Arc the Radius will always be same. Hence a Chord of the Circular Arc always cut an Isosceles Triangle with the two equal sides, equal to the Radius.

Determining the Subtended Angle " $\square$ ' at the Center of the Circular Arc

From our Conjure the Equivalent Circular Arc will cut an angle equal to the difference between the parametric values of the end points of the Circular Arc. Let this Angle be ' $\square$ '.

Then ' $\square$ ' is calculated as

$$
\square=t_{1}-t_{2}
$$

Determining the Radius of the Circular Arc

In order to determine the Radius of the Circular Arc we now define the isosceles triangle formed by the two radiuses of the Circular Arc and the Chord of the Circular Arc.

From the Conjure, the Circular Arc's Chord length is the same as that of the Elliptical Arc. Hence we know the base length ' $\boldsymbol{h}$ ' of the Isosceles Triangle as length of AB. Also we know the angle opposite to the base of the Isosceles triangle as ' $\square$ ' from the Conjure. 
We can now algebraically determine the length of the side of the Isosceles Triangle denoted ' $\mathbf{R}_{\mathbf{c}}$ ' which will be equal to the Radius of the Circular Arc using the formula

$$
R_{c}=\frac{h}{2 \sin \left(\frac{\theta}{2}\right)}
$$

Where ' $\boldsymbol{h}$ ' is the Chord length and ' $\square$ ' is the angle subtended opposite to the base.

For derivation; Reference: Appendix A

\section{Determine the Arc length}

We now have the Radius and Subtended angle of the Circular Arc. We can now define the Circular Arc

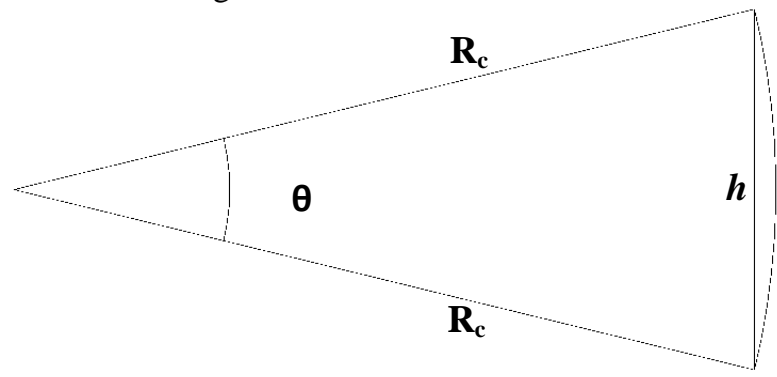

(Fig 2)

\section{Result}

Once we have defined the Circular Arc whose Arc length is equal to the Elliptical Arc we can determine the Elliptical Arc length ' $\boldsymbol{L}_{\boldsymbol{e}}$ ' using the Arc length formula for Circular Arc as

\section{Elliptical Arc Length $\left(L_{e}\right)=\mathbf{R}_{\mathrm{c}} . \theta$}

Substituting for ' $\mathbf{R}_{\mathbf{c}}$ ', we get the Equation in single form as

$$
\text { Elliptical Arc Length }\left(L_{e}\right)=\left\{\frac{h}{2 \sin \left(\frac{\theta}{2}\right)}\right\} . \theta
$$

Substituting for ' $\boldsymbol{h}$ ' and ' $\square$ ' we get the formulae in terms of the coordinates of the end points of the Elliptical Arc and the parametric values of the End points of the Elliptical Arc as

$$
\text { Elliptical Arc Length }\left(L_{e}\right)=\left\{\frac{\sqrt{\left(x_{2}-x_{1}\right)^{2}+\left(y_{2}-y_{1}\right)^{2}}}{2 \sin \left(\frac{t_{1}-t_{2}}{2}\right)}\right\} \cdot\left\{t_{1}-t_{2}\right\}
$$

\section{Conclusions}

Thus we have a formula in standard trigonometric functions to determine the Arc length of given Elliptical Arc segment lying within a Quadrant of the Ellipse.

Suppose the Major and Minor axis lengths are made equal the Ellipse reduces to a circle. In this case the derived formula reduces to the standard Arc length formula for the Circular Arc.

[1] Wikipedia Article: Ellipse

\section{References}

[2] Wikipedia Article: Elliptic integral

[3] Wikipedia Article: Trigonometric functions:

[4] Wikipedia Article: Eccentric Anomaly

[5] Wikipedia Article: Elliptical Integral

[6] Appendices

[7] Appendix A: Determine the side of an Isosceles Triangle given the Angle opposite to the Base and Base length

[8] Appendix B: Approximating the Incomplete Elliptical Integral of Second Kind

[9] Appendix C: Sample Problem \& Solution Algorithm

Appendix A: Determine the Side of an Isosceles Triangle given the Angle opposite to the Base and Base length 
Consider the Isosceles triangle PQR. Let the Base length 'QR' be given as ' $\boldsymbol{h}$ ' and the length of the equal Sides 'PQ' \& 'PR' be given as ' $\mathbf{R}_{c}$ '. Let the angle RPQ be given as ' $\square$ '. Then the equal angles 'PQR' \& 'QRP' are given as $\left(\frac{\pi-\theta}{2}\right)$, (Since sum of the angles of a triangle is equal to ' $\pi$ ').

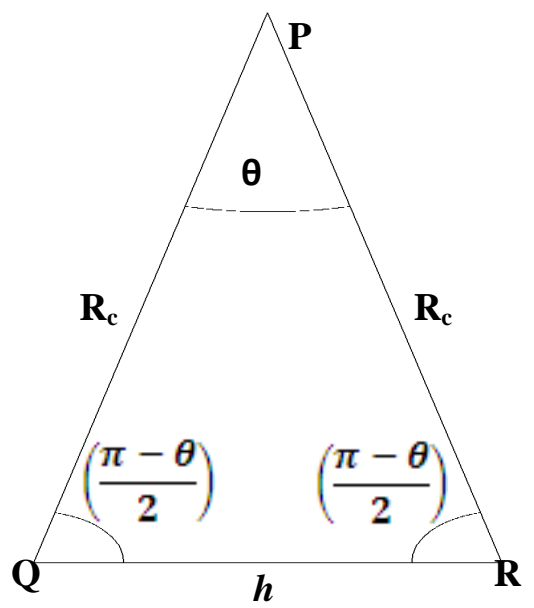

From Law of Sines we get

$\frac{R_{c}}{\sin \left\{\frac{(\pi-\theta)}{2}\right\}}=\frac{R_{c}}{\sin \left\{\frac{(\pi-\theta)}{2}\right\}}=\frac{h}{\sin \theta} \quad--------(1)$

From Equation (1) Consider the Identity

$\frac{R_{c}}{\sin \left\{\frac{(\pi-\theta)}{2}\right\}}=\frac{h}{\sin \theta}$

Let us analyze

$\sin \left\{\frac{(\pi-\theta)}{2}\right\}=\sin \left(\frac{\pi}{2}-\frac{\theta}{2}\right)$

Consider the trigonometric identity

$\sin \left(\frac{\pi}{2}-A\right)=\cos A$

(The Angle ' $A$ ' is in Radians)

Applying the identity to Equation (3) we get

$\sin \left\{\frac{(\pi-\theta)}{2}\right\}=\cos \frac{\theta}{2}$

Substituting result (4) in Equation (2) we get

$$
\frac{R_{c}}{\cos \frac{\theta}{2}}=\frac{h}{\sin \theta}
$$

We know from the Double Angle Formulae

$$
\sin 2 A=2 \sin A \cos A
$$

Applying this to $\sin \theta$

$$
\begin{aligned}
& \sin \theta=\sin \left\{2\left(\frac{\theta}{2}\right)\right\} \\
& \sin \left\{2\left(\frac{\theta}{2}\right)\right\}=2 \sin \frac{\theta}{2} \cos \frac{\theta}{2}
\end{aligned}
$$

Substituting result (6) in Equation (5) we get 


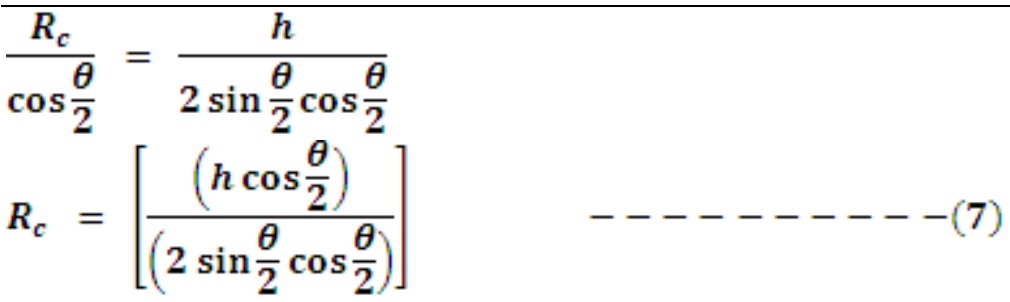

$\underline{\text { Result }}$

$\overline{R_{c}}=\frac{h}{\left(2 \sin \frac{\theta}{2}\right)}$

\section{Appendix B: Approximating the Incomplete Elliptical Integral of Second Kind}

The Elliptical Integral giving the Arc length of the Elliptical Arc segment in trigonometric form is given as

$$
L_{e}=b \int_{t_{\mathrm{n}}}^{t_{1}} \sqrt{\left[1-e^{2} \sin (t)^{2}\right]} d t
$$

Where ' $\boldsymbol{e}$ ' is the Eccentricity of the Ellipse, the current known solution to the integral is through numerical Integration. A method to estimate a closed form solution to this integral using the geometric method is detailed below.

\section{Approximating the Incomplete Elliptical Integral of Second Kind}

From the Geometric method determining the Arc Length, we can estimate the incomplete Elliptical Integral as well as the Complete Elliptical Integral of the Second Kind. In our method of determining the Arc Length we transform the Elliptical Arc to an equivalent Circular Arc with the same Arc length. Let the Elliptical Arc length be represented ' $L_{e}$ ' and the Circular Arc length be ' $L_{c}$ '. The transformation in integral form can be written as,

$$
\begin{gathered}
L_{e} \approx L_{c} \\
L_{e}=b \int_{t_{2}}^{t_{1}} \sqrt{\left(1-e^{2} \sin (t)^{2}\right.} d t \approx \int_{t_{2}}^{t_{1}} R(t) d t=L_{c} \\
L_{c}=\int_{t_{2}}^{t_{1}} R(t) d t=R(t) \int_{t_{2}}^{t_{1}} d t=R(t)\left(t_{1}-t_{2}\right)
\end{gathered}
$$

Then

$$
b \int_{t_{2}}^{t_{1}} \sqrt{\left(1-e^{2} \sin (t)^{2}\right.} d t \approx \int_{t_{2}}^{t_{11}} R(\mathrm{t}) d t=R(t) \cdot\left(t_{1}-t_{2}\right)
$$

Result

$$
b \int_{t_{2}}^{t_{1}} \sqrt{\left[1-e^{2} \sin (t)^{2}\right]} d t \approx \mathrm{R}(\mathrm{t}) \cdot\left(t_{1}-t_{2}\right)
$$

Analyzing the geometric intuition the Radius, ' $R(t)$ ' is given as

$$
\boldsymbol{R}(\boldsymbol{t})=\left\{\frac{\sqrt{\left(x_{2}-x_{1}\right)^{2}+\left(y_{2}-y_{1}\right)^{2}}}{2 \sin \left(\frac{t_{1}-t_{2}}{2}\right)}\right\}
$$

Where $\boldsymbol{x}_{1}, \boldsymbol{y}_{1}, \boldsymbol{x}_{2}, \& \boldsymbol{y}_{2}$ is given as

$$
\begin{gathered}
x_{1}=\boldsymbol{a} \operatorname{Cos}\left(t_{1}\right), \quad y_{1}=\boldsymbol{b} \operatorname{Sin}\left(t_{1}\right) \& \\
x_{2}=\boldsymbol{a} \operatorname{Cos}\left(t_{2}\right), \quad y_{2}=\boldsymbol{b} \operatorname{Sin}\left(t_{2}\right)
\end{gathered}
$$

Where ' $\mathbf{a}$ ' $\&$ ' $\mathbf{b}$ ' are Constants (i.e. semi-major and semi-minor axis of ellipse), and ' $\boldsymbol{t}_{\boldsymbol{1}}$ ' $\&$ ' $\boldsymbol{t} \boldsymbol{t}_{2}$ ' are the limits of the Incomplete, Elliptical Integral of Second kind.

\section{Solution}

The Closed form solution in Single form is given as

$$
b \int_{t_{2}}^{t_{1}} \sqrt{\left[1-e^{2} \sin (t)^{2}\right]} d t=\left\{\frac{\sqrt{\left(a \cos t_{2}-a \cos t_{1}\right)^{2}+\left(b \sin t_{2}-b \sin t_{1}\right)^{2}}}{2 \sin \left(\frac{t_{1}-t_{2}}{2}\right)}\right\}\left\{t_{1}-t_{2}\right\}
$$


$b \int_{t_{2}}^{t_{1}} \sqrt{\left[1-e^{2} \sin (t)^{2}\right]} d t=\left\{\frac{\sqrt{a^{2}\left(\cos t_{2}-\cos t_{1}\right)^{2}+b^{2}\left(\sin t_{2}-\sin t_{1}\right)^{2}}}{2 \sin \left(\frac{t_{1}-t_{2}}{2}\right)}\right\}\left\{t_{1}-t_{2}\right\}$

Conclusion and Future Prospects

Developing an analytical, algebraic theory to explain the geometric result based on the geometric intuition can be a precursor to developing a method of solving the Elliptical Integrals. The Method can be further refined to encompass the whole class of functions defined as the Elliptic function. The geometric fact certifies an analogy

\section{Appendix C: Sample Problem \& Solution Algorithm \\ Problem Description}

It is required to calculate the Arc Length $\left(L_{e}\right)$ of an Elliptical Arc (within a Quadrant of the Ellipse) given the Coordinates of the End points of the Arc. Let the End points be designated ' $A$ ' \& 'B' and the Coordinates be $\left(\boldsymbol{x}_{1}, \boldsymbol{y}_{1}\right)$ and $\left(\boldsymbol{x}_{2}, \boldsymbol{y}_{2}\right)$ respectively.

The Semi Major Axis and Semi Minor Axis are 'a' and 'b' respectively. The Ellipse is aligned in the Canonical Position i.e. center at origin and Major axis along the $\mathrm{x}$ - axis.

Solution Algorithm

$\underline{\text { Step 1: }}$ Determine the parametric value of the end points of the given Arc

Let the Parametric value (Eccentric Anomaly) be given as ' $t_{1}$ ' for point ' $\mathbf{A}$ : $\left(\boldsymbol{x}_{1}, \boldsymbol{y}_{1}\right)$ ' and ' $\boldsymbol{t}_{2}$ ' for point 'B: $\left(\boldsymbol{x}_{2}\right.$, $\left.y_{2}\right)^{\prime}$.

The parametric values are then determined as

$$
\begin{aligned}
& x_{1}=a \cos \left(t_{1}\right) \\
& \cos \left(t_{1}\right)=\frac{x_{1}}{a} \\
& t_{1}=\cos ^{-1}\left(\frac{x_{1}}{a}\right) \\
& x_{2}=a \cos \left(t_{2}\right) \\
& \cos \left(t_{2}\right)=\frac{x_{2}}{a} \\
& t_{2}=\cos ^{-1}\left(\frac{x_{2}}{a}\right)
\end{aligned}
$$

Step 2: Determine the Subtended angle ' $\square$ ' of the Equivalent Circular Arc

The subtended angle has a value in radians equal to the difference between the parametric values (Eccentric Anomalies) of the End points of the Given Arc, i.e. ' $\boldsymbol{t}_{\boldsymbol{1}}$ ' for Point ' $\mathbf{A}$ ' \& ' $\boldsymbol{t}_{2}$ ' for Point ' $\mathbf{B}$ ' The value of ' $\square$ ' in Radians is determined as

$$
\square=t_{1}-t_{2}
$$

Step 3: Verify the $y$-coordinate values of the end points ' $\mathbf{A}$ ' \& ' $\mathbf{B}$ ' of the Elliptical Arc as

$y_{1}=b \sin t_{1}$

$y_{2}=b \sin t_{2}$

Step 4: Determine Length of the Chord of the Equivalent Circular Arc ' $\boldsymbol{h}$ '

The Chord of the Equivalent Circular Arc will have the same Arc length as that of the Chord of the Elliptical Arc. Since they have the same length let us denote the Chord of the Elliptical Arc also as ' $\boldsymbol{h}$ '. The length ' $\boldsymbol{h}$ ' is then given as

$$
h=\sqrt{\Delta x^{2}+\Delta y^{2}}
$$

$$
h=\sqrt{\left(x_{2}-x_{1}\right)^{2}+\left(y_{2}-y_{1}\right)^{2}}
$$

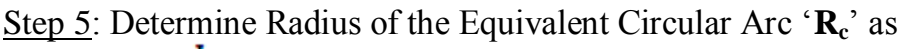

$R_{c}=\frac{h}{\left(2 \sin \frac{\theta}{2}\right)}$

Step 6: Determine Arc Length of the given Arc Elliptical Arc ' $L_{e}$ ' as

Elliptical Arc Length $\left(L_{e}\right)=\mathbf{R}_{\mathrm{c}} \cdot \theta$ 